\title{
The Elliptic Sinh-Gordon Equation in the Quarter Plane
}

Guenbo Hwang

To cite this article: Guenbo Hwang (2016) The Elliptic Sinh-Gordon Equation in the Quarter Plane, Journal of Nonlinear Mathematical Physics 23:1, 127-140, DOI: https://doi.org/10.1080/14029251.2016.1135646

To link to this article: https://doi.org/10.1080/14029251.2016.1135646

Published online: 04 January 2021 


\title{
The Elliptic Sinh-Gordon Equation in the Quarter Plane
}

\author{
Guenbo Hwang \\ Department of Mathematics, Daegu University, \\ Gyeongsan Gyeongbuk, 712-714, Korea \\ ghwang@daegu.ac.kr
}

Received 10 August 2015

Accepted 20 November 2015

\begin{abstract}
We study the elliptic sinh-Gordon equation formulated in the quarter plane by using the so-called Fokas method, which is a significant extension of the inverse scattering transform for the boundary value problems. The method is based on the simultaneous spectral analysis for both parts of the Lax pair and the global algebraic relation that involves all boundary values. In this paper, we address the existence theorem for the elliptic sinh-Gordon equation posed in the quarter plane under the assumption that the boundary values satisfy the global relation. We also present the formal representation of the solution in terms of the unique solution of the matrix RiemannHilbert problem defined by the spectral functions.
\end{abstract}

Keywords: Boundary value problem; Integrable system; Sinh-Gordon equation.

2000 Mathematics Subject Classification: 47K15, 35Q55

\section{Introduction}

We study the boundary problem for the elliptic sinh-Gordon equation posed in the quarter plane

$$
q_{x x}+q_{y y}=\sinh q, \quad(x, y) \in \Omega,
$$

where $\Omega=\left\{(x, y) \in \mathbb{R}^{2}: 0<x<\infty, 0<y<\infty\right\}$. It is noted that this equation arises as models of interacting charged particles in plasma physics [16]. On the other hand, of interest is the integrability for the equation; the elliptic sinh-Gordon equation is a reduction of the special case of the Toda lattice equations [1]. As a consequence, the usual inverse scattering transform can be used to solve the elliptic sinh-Gordon equation in the entire plane $\{-\infty<x, y<\infty\}[3,16]$. Regarding more complicated domains, the so-called Fokas method is remarkably elegant for solving boundary value problems. The method is widely used to analyze a large class of partial differential equations and hence, it can be considered as a significant generalization of the inverse scattering transform $[2,4,5$, 7] (see also the monograph [9] and recent applications [10,15]). Recently, the elliptic sinh-Gordon equation posed in the half plane $\{-\infty<x<\infty, 0<y<\infty\}$ was studied by applying the Fokas method [14]. It has been shown that the solution of the equation in the half plane exists provided that the boundary values satisfy the global algebraic relation that is the simple but substantial equation involving all boundary values. In the implementation of the Fokas method, the global relation is crucial in proving the existence of the unique solution and characterizing unknown boundary values called the generalized Dirichlet to Neumann map [8].

In this paper, we implement the Fokas method to analyze the elliptic sinh-Gordon equation posed in the quarter plane (1.1). Based on the spectral analysis in the Lax pair, we derive global relation in terms of the spectral functions. We then formulate the matrix Riemann-Hilbert problem 
with the jump matrices defined uniquely by the spectral functions. These spectral functions denoted by $\left\{a_{1}(k), b_{1}(k)\right\}$ and $\left\{a_{2}(k), b_{2}(k)\right\}$ can be determined from the boundary values $\left\{q(x, 0), q_{y}(x, 0)\right\}$ and $\left\{q(0, y), q_{x}(0, y)\right\}$, respectively. Moreover, we show that the given boundary values with appropriate regularity condition, the solution for (1.1) uniquely exists if the boundary values satisfy the global relation. In addition to the existence of the solution, we address the formal representation for the solution in terms of the unique solution of the Riemann-Hilbert problem (see $[14,17,18]$ for analogous results).

The outline of the paper is following. In section 2, we introduce the Lax pair for the elliptic sinhGordon equation and the regularity assumption for the boundary values as well as relevant notations and formulas. In section 3, we derive the global relation and we then define spectral functions that determine the jump matrices for the Riemann-Hilbert problem. Moreover, we apply the spectral analysis for the Lax pair at $x=0$ and $y=0$ in order to characterize the boundary values. In section 4 , the existence of the solution is discussed by analyzing the the matrix Riemann-Hilbert problem as an inverse problem. We end with concluding remarks in section 5 .

\section{Preliminaries}

It is well known that the elliptic sinh-Gordon equation (1.1) can be written as an overdetermined linear system called a Lax pair $[3,14,16]$

$$
\begin{aligned}
& \mu_{x}+\omega_{1}(k)\left[\sigma_{3}, \mu\right]=Q(x, y, k) \mu, \\
& \mu_{y}+\omega_{2}(k)\left[\sigma_{3}, \mu\right]=i \tilde{Q}(x, y, k) \mu
\end{aligned}
$$

where $k \in \mathbb{C}$ is a spectral parameter, $\mu$ is a $2 \times 2$ matrix-valued eigenfunction and

$$
\begin{aligned}
& \omega_{1}(k)=-\frac{1}{2 i}\left(k-\frac{1}{4 k}\right), \quad \omega_{2}(k)=-\frac{1}{2}\left(k+\frac{1}{4 k}\right), \\
& Q(x, y, k)=\frac{1}{4}\left(\begin{array}{cc}
\frac{i}{2 k}(\cosh q-1) & -\left(r+\frac{\sinh q}{2 k}\right) \\
r-\frac{\sinh q}{2 k} & -\frac{i}{2 k}(\cosh q-1)
\end{array}\right)
\end{aligned}
$$

with

$$
\begin{aligned}
& \tilde{Q}(x, y, k)=Q(x, y,-k), \\
& r(x, y)=i q_{x}(x, y)+q_{y}(x, y), \quad \sigma_{3}=\left(\begin{array}{cc}
1 & 0 \\
0 & -1
\end{array}\right)
\end{aligned}
$$

and the matrix commutator given by $\left[\sigma_{3}, A\right]=\sigma_{3} A-A \sigma_{3}$.

Note that the elliptic sinh-Gordon equation (1.1) is the compatible condition for the Lax pair (2.1); $\mu_{x y}=\mu_{y x}$ in the Lax pair (2.1) implies that the function $q(x, y)$ solves (1.1) provided that the spectral parameter $k$ is independent of $x$ and $y$. From the definitions of $\omega_{1}(k)$ and $\omega_{2}(k)$, it follows that

$$
\operatorname{Re} \omega_{1}(k)=-\frac{1}{8}\left(4+\frac{1}{|k|^{2}}\right) \operatorname{Im} k, \quad \operatorname{Re} \omega_{2}(k)=-\frac{1}{8}\left(4+\frac{1}{|k|^{2}}\right) \operatorname{Re} k
$$

Thus, we find

$$
\operatorname{Re} \omega_{1}(k)<0 \quad \text { for } \operatorname{Im} k>0, \quad \operatorname{Re} \omega_{2}(k)<0 \quad \text { for } \operatorname{Re} k>0
$$




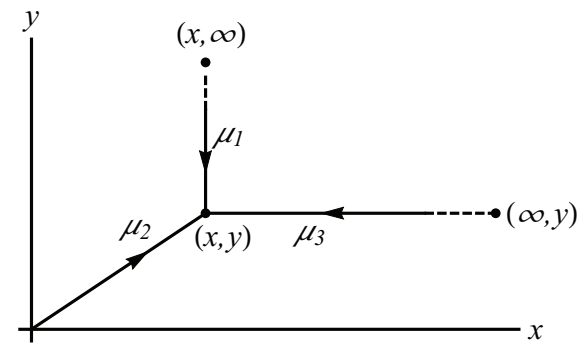

Fig. 1. The three distinct points for the definition of the eigenfunction $\mu_{j}, j=1,2,3$

Due to the symmetry of $Q$ and $\tilde{Q}$, the eigenfunction possesses the same symmetry, namely,

$$
\mu_{11}(x, y, k)=\mu_{22}(x, y,-k), \quad \mu_{21}(x, y, k)=-\mu_{12}(x, y,-k),
$$

where the subscripts denote the $(i, j)$-component of the matrix.

It is convenient to introduce the notation $\hat{\sigma} A=\left[\sigma_{3}, A\right]$ for the matrix commutator. Using this notation, we let the following notation

$$
e^{\hat{\sigma}_{3} \xi} A=e^{\sigma_{3} \xi} A e^{-\sigma_{3} \xi}=\left(\begin{array}{cc}
a_{11} & e^{2 \xi} a_{12} \\
e^{-2 \xi} a_{21} & a_{22}
\end{array}\right)
$$

We also denote boundary values by

$$
\begin{array}{ll}
q(x, 0)=g_{0}(x), & q_{y}(x, 0)=g_{1}(x), \\
q(0, y)=f_{0}(y), & q_{x}(0, y)=f_{1}(y),
\end{array}
$$

where we assume that $g_{j}, f_{j} \in H^{1}\left(\mathbb{R}^{+}\right)$for $j=0,1$.

In order to analyze the Lax pair (2.1), we first define a differential 1-form $W$ given by

$$
W(x, y, k)=Q(x, y, k) \mu(x, y, k) d x+i \tilde{Q}(x, y, k) \mu(x, y, k) d y,
$$

which implies that (2.1) is equivalent to the form

$$
d\left[e^{\left(\omega_{1}(k) x+\omega_{2}(k) y\right) \hat{\sigma}_{3}} \mu(x, y, k)\right]=e^{\left(\omega_{1}(k) x+\omega_{2}(k) y\right) \hat{\sigma}_{3}} W(x, y, k) .
$$

Hence, we define eigenfunctions that satisfy both parts of the Lax pair (2.1) as

$$
\mu_{j}(x, y, k)=I+\int_{\left(x_{j}, y_{j}\right)}^{(x, y)} e^{-\left(\omega_{1}(k)(x-\xi)+\omega_{2}(k)(y-\eta)\right) \hat{\sigma}_{3}} W_{j}(\xi, \eta, k),
$$

where $(x, y),\left(x_{j}, y_{j}\right) \in \Omega=\{0<x<\infty, 0<y<\infty\}$ and $W_{j}$ is the differential form defined by (2.9) with $\mu_{j}$. Since the differential 1-form $W(x, y, k)$ is closed, the integration in (2.10) does not depend 
on paths [6]. In particular, we choose three distinct points $\left(x_{j}, y_{j}\right)$ in $\Omega, j=1,2,3$ (see Fig. 1),

$$
\left(x_{1}, y_{1}\right)=(x, \infty), \quad\left(x_{2}, y_{2}\right)=(0,0), \quad\left(x_{3}, y_{3}\right)=(\infty, y) .
$$

More specifically, the eigenfunctions associated with the points $\left(x_{j}, y_{j}\right), j=1,2,3$, satisfy the following integral equations:

$$
\begin{aligned}
\mu_{1}(x, y, k)= & I-i \int_{y}^{\infty} e^{-\omega_{2}(k)(y-\eta) \hat{\sigma}_{3}}\left(\tilde{Q} \mu_{1}\right)(x, \eta, k) d \eta \\
\mu_{2}(x, y, k)= & I+\int_{0}^{x} e^{-\omega_{1}(k)(x-\xi) \hat{\sigma}_{3}}\left(Q \mu_{2}\right)(\xi, y, k) d \xi \\
& +i \int_{0}^{y} e^{-\left(\omega_{1}(k) x+\omega_{2}(k)(y-\eta)\right) \hat{\sigma}_{3}}\left(\tilde{Q} \mu_{2}\right)(0, \eta, k) d \eta, \\
\mu_{3}(x, y, k)= & I-\int_{x}^{\infty} e^{-\omega_{1}(k)(x-\xi) \hat{\sigma}_{3}}\left(Q \mu_{3}\right)(\xi, y, k) d \xi .
\end{aligned}
$$

It should be remarked that the off-diagonal components of the matrix-valued eigenfunctions $\mu_{j}$ involve the explicit exponential terms. Thus, according to equations (2.6), let the domains $D_{j}$ in the complex $k$-plane, $j=1, \cdots, 4$, be depicted in Fig. 2 and be defined by

$$
\begin{aligned}
& D_{1}=\left\{k \in \mathbb{C}: \operatorname{Re} \omega_{1}(k)<0\right\} \cap\left\{k \in \mathbb{C}: \operatorname{Re} \omega_{2}(k)<0\right\}, \\
& D_{2}=\left\{k \in \mathbb{C}: \operatorname{Re} \omega_{1}(k)<0\right\} \cap\left\{k \in \mathbb{C}: \operatorname{Re} \omega_{2}(k)>0\right\}, \\
& D_{3}=\left\{k \in \mathbb{C}: \operatorname{Re} \omega_{1}(k)>0\right\} \cap\left\{k \in \mathbb{C}: \operatorname{Re} \omega_{2}(k)>0\right\}, \\
& D_{4}=\left\{k \in \mathbb{C}: \operatorname{Re} \omega_{1}(k)>0\right\} \cap\left\{k \in \mathbb{C}: \operatorname{Re} \omega_{2}(k)<0\right\} .
\end{aligned}
$$

As a result, the domains of analyticity and boundedness for the eigenfunctions can be determined:

- $\mu_{1}(x, y, k)$ is analytic and bounded for $k \in\left(D_{2} \cup D_{3}, D_{1} \cup D_{4}\right)$,

- $\mu_{2}(x, y, k)$ is analytic and bounded for $k \in\left(D_{1}, D_{3}\right)$,

- $\mu_{3}(x, y, k)$ is analytic and bounded for $k \in\left(D_{3} \cup D_{4}, D_{1} \cup D_{2}\right)$.

For convenience, we write each column of $\mu_{j}(x, y, k)$ as the following notations:

$$
\mu_{1}=\left(\mu_{1}^{(23)}, \mu_{1}^{(14)}\right), \quad \mu_{2}=\left(\mu_{2}^{(1)}, \mu_{2}^{(3)}\right), \quad \mu_{3}=\left(\mu_{3}^{(34)}, \mu_{3}^{(12)}\right),
$$

where the superscripts indicate the analytic and bounded domains $D_{j}, j=1, \cdots, 4$, for the columns of the matrix-valued eigenfunctions. Using integration by parts, note that in the appropriate domain

$$
\mu_{j}(x, y, k)=I+O(1 / k) \quad \text { as } \quad k \rightarrow \infty .
$$

Since the matrices $Q$ and $\tilde{Q}$ are traceless (i.e. $\operatorname{trace}(Q)=\operatorname{trace}(\tilde{Q})=0)$, equation (2.13) implies that $\operatorname{det} \mu_{j}=1, j=1,2,3$.

\section{Spectral analysis}

\subsection{Spectral functions}

The matrix eigenfunctions $\mu_{1}, \mu_{2}$ and $\mu_{3}$ are both fundamental solutions of the Lax pair (2.1). Note that $\mu_{2}(0,0, k)=I$. Hence, the eigenfunctions are related by the so-called spectral functions, also 
G. Hwang / The Elliptic Sinh-Gordon Equation in the Quarter Plane

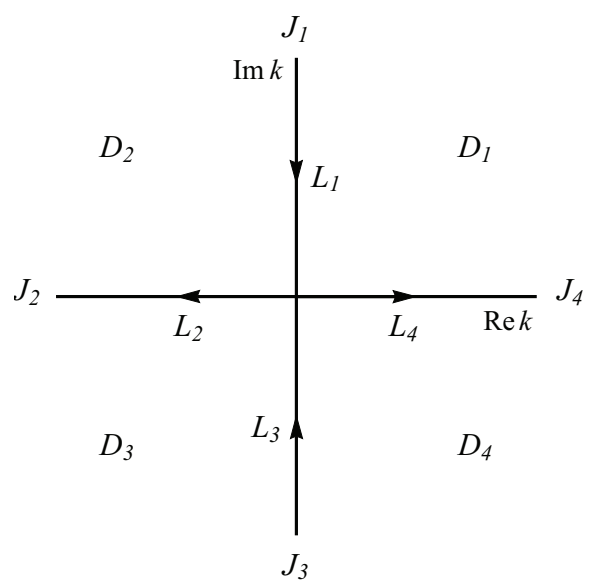

Fig. 2. The domain $D_{j}$ and the oriented contour $L_{j}, j=1, \cdots, 4$

known as the scattering matrices, $S_{1}(k)$ and $S_{2}(k)$ :

$$
\begin{array}{ll}
\mu_{3}(x, y, k)=\mu_{2}(x, y, k) e^{-\left(\omega_{1}(k) x+\omega_{2}(k) y\right) \hat{\sigma}_{3}} S_{1}(k), & k \in\left(\mathbb{R}^{+}, \mathbb{R}^{-}\right), \quad 0 \leq x, y<\infty, \\
\mu_{1}(x, y, k)=\mu_{2}(x, y, k) e^{-\left(\omega_{1}(k) x+\omega_{2}(k) y\right) \hat{\sigma}_{3}} S_{2}(k), & k \in\left(i \mathbb{R}^{+}, i \mathbb{R}^{-}\right), \quad 0 \leq x, y<\infty .
\end{array}
$$

Substituting (3.1b) into (3.1a), of course, equations (3.1) are determined in the form

$$
\mu_{3}(x, y, k)=\mu_{1}(x, y, k) e^{-\left(\omega_{1}(k) x+\omega_{2}(k) y\right) \hat{\sigma}_{3}}\left(S_{2}^{-1}(k) S_{1}(k)\right), \quad k \in\left(\partial D_{1}, \partial D_{3}\right) .
$$

Letting $x=0$ and $y=0$ in (3.1), the spectral functions are given by

$$
S_{1}(k)=\mu_{3}(0,0, k), \quad S_{2}(k)=\mu_{1}(0,0, k) .
$$

From the symmetry (2.7) of the eigenfunctions $\mu_{1}$ and $\mu_{3}$, we write the spectral functions $S_{1}(k)$ and $S_{2}(k)$ as

$$
S_{1}(k)=\left(\begin{array}{cc}
a_{1}(k) & -b_{1}(-k) \\
b_{1}(k) & a_{1}(-k)
\end{array}\right), \quad S_{2}(k)=\left(\begin{array}{cc}
a_{2}(k) & -b_{2}(-k) \\
b_{2}(k) & a_{2}(-k)
\end{array}\right) .
$$

Since $\operatorname{det} S_{1}(k)=\operatorname{det} S_{2}(k)=1$, we find the identites

$$
a_{1}(k) a_{1}(-k)+b_{1}(k) b_{1}(-k)=1, \quad a_{2}(k) a_{2}(-k)+b_{2}(k) b_{2}(-k)=1 .
$$

Furthermore, we define

$$
\Phi(x, k)=\mu_{3}(x, 0, k), \quad \Psi(y, k)=\mu_{1}(0, y, k),
$$

that is, the functions $\Phi$ and $\Psi$ satisfy the following integral equations

$$
\begin{aligned}
& \Phi(x, k)=I-\int_{x}^{\infty} e^{-\omega_{1}(k)(x-\xi) \hat{\sigma}_{3}}\left(Q_{0} \Phi\right)(\xi, k) d \xi, \quad k \in\left(D_{1} \cup D_{2}, D_{3} \cup D_{4}\right), \quad 0 \leq x<\infty, \\
& \Psi(y, k)=I-i \int_{y}^{\infty} e^{-\omega_{2}(k)(y-\eta) \hat{\sigma}_{3}}\left(\tilde{Q}_{0} \Psi\right)(\eta, k) d \eta, \quad k \in\left(D_{2} \cup D_{3}, D_{1} \cup D_{4}\right), \quad 0 \leq y<\infty,
\end{aligned}
$$


where $Q_{0}(x, k)=Q(x, 0, k)$ and $\tilde{Q}_{0}(y, k)=Q(0, y,-k)$. Note that

$$
S_{1}(k)=\Phi(0, k), \quad S_{2}(k)=\Psi(0, k),
$$

which immediately imply that the spectral functions $a_{1}(k)$ and $b_{1}(k)$ have analytic continuations for $\operatorname{Im} k<0$, while the spectral functions $a_{2}(k)$ and $b_{2}(k)$ have analytic continuations for $\operatorname{Re} k<0$. Moreover, due to the symmetry (2.7), the functions $\Phi$ and $\Psi$ also can be written as

$$
\Phi(x, k)=\left(\begin{array}{cc}
\Phi_{1}(x, k) & -\Phi_{2}(x,-k) \\
\Phi_{2}(x, k) & \Phi_{1}(x,-k)
\end{array}\right), \quad \Psi(y, k)=\left(\begin{array}{cc}
\Psi_{1}(y, k) & -\Psi_{2}(y,-k) \\
\Psi_{2}(x, k) & \Psi_{1}(y,-k)
\end{array}\right)
$$

We now define the integral representations for the spectral functions below.

Definition 3.1. Given $q(x, 0)=g_{0}(x)$ and $q_{y}(x, 0)=g_{1}(x)$, the map

$$
\left\{g_{0}(x), g_{1}(x)\right\} \rightarrow\left\{a_{1}(k), b_{1}(k)\right\}
$$

is defined by

$$
\begin{aligned}
a_{1}(k)= & -\frac{1}{4} \int_{0}^{\infty}\left\{\frac{i}{2 k}\left(\cosh g_{0}(\xi)-1\right) \Phi_{1}(\xi, k)\right. \\
& \left.-\left(i \dot{g}_{0}(\xi)+g_{1}(\xi)+\frac{\sinh g_{0}(\xi)}{2 k}\right) \Phi_{2}(\xi, k)\right\} d \xi, \quad \operatorname{Im} k<0, \\
b_{1}(k)= & -\frac{1}{4} \int_{0}^{\infty} e^{-2 \omega_{1}(k) \xi}\left\{\left(i \dot{g}_{0}(\xi)+g_{1}(\xi)-\frac{\sinh g_{0}(\xi)}{2 k}\right) \Phi_{1}(\xi, k)\right. \\
& \left.-\frac{i}{2 k}\left(\cosh g_{0}(\xi)-1\right) \Phi_{2}(\xi, k)\right\} d \xi, \quad \operatorname{Im} k<0,
\end{aligned}
$$

where the functions $\Phi_{1}$ and $\Phi_{2}$ are solutions of the $x$-part of the Lax pair (2.1a) with $y=0$, that is, $\Phi_{1}$ and $\Phi_{2}$ solve the following system of ordinary differential equations:

$$
\begin{aligned}
& \Phi_{1 x}=\frac{1}{4}\left[\frac{i}{2 k}\left(\cosh g_{0}(x)-1\right) \Phi_{1}-\left(i \dot{g}_{0}(x)+g_{1}(x)+\frac{\sinh g_{0}(x)}{2 k}\right) \Phi_{2}\right], \\
& \Phi_{2 x}-2 \omega_{1}(k) \Phi_{2}=\frac{1}{4}\left[\left(i \dot{g}_{0}(x)+g_{1}(x)-\frac{\sinh g_{0}(x)}{2 k}\right) \Phi_{1}-\frac{i}{2 k}\left(\cosh g_{0}(x)-1\right) \Phi_{2}\right]
\end{aligned}
$$

with $\lim _{x \rightarrow \infty}\left(\Phi_{1}, \Phi_{2}\right)=(1,0)$.

Definition 3.2. Given $q(0, y)=f_{0}(y)$ and $q_{x}(0, y)=f_{1}(y)$, the map

$$
\left\{f_{0}(y), f_{1}(x)\right\} \rightarrow\left\{a_{2}(k), b_{2}(k)\right\}
$$


is defined by

$$
\begin{aligned}
a_{2}(k)= & -\frac{i}{4} \int_{0}^{\infty}\left\{-\frac{i}{2 k}\left(\cosh f_{0}(\eta)-1\right) \Psi_{1}(\eta, k)\right. \\
& \left.-\left(i f_{1}(\eta)+\dot{f}_{0}(\eta)-\frac{\sinh f_{0}(\eta)}{2 k}\right) \Psi_{2}(\eta, k)\right\} d \eta, \quad \operatorname{Re} k<0, \\
b_{2}(k)= & -\frac{i}{4} \int_{0}^{\infty} e^{-2 \omega_{2}(k) \eta}\left\{\left(i f_{1}(\eta)+\dot{f}_{0}(\eta)+\frac{\sinh f_{0}(\xi)}{2 k}\right) \Psi_{1}(\eta, k)\right. \\
& \left.+\frac{i}{2 k}\left(\cosh f_{0}(\eta)-1\right) \Psi_{2}(\eta, k)\right\} d \eta, \quad \operatorname{Re} k<0,
\end{aligned}
$$

where the functions $\Psi_{1}$ and $\Psi_{2}$ are solutions of the $y$-part of the Lax pair (2.1b) with $x=0$, that is, $\Psi_{1}$ and $\Psi_{2}$ solve the following system of ordinary differential equations:

$$
\begin{aligned}
& \Psi_{1 y}=\frac{i}{4}\left[-\frac{i}{2 k}\left(\cosh f_{0}(y)-1\right) \Psi_{1}-\left(i f_{0}(y)+\dot{f}_{1}(y)-\frac{\sinh f_{0}(y)}{2 k}\right) \Psi_{2}\right], \\
& \Psi_{2 y}-2 \omega_{2}(k) \Psi_{2}=\frac{i}{4}\left[\left(i f_{0}(y)+\dot{f}_{1}(y)+\frac{\sinh f_{0}(y)}{2 k}\right) \Psi_{1}+\frac{i}{2 k}\left(\cosh f_{0}(y)-1\right) \Psi_{2}\right]
\end{aligned}
$$

with $\lim _{y \rightarrow \infty}\left(\Psi_{1}, \Psi_{2}\right)=(1,0)$.

In what follows we derive the global relation which is the key to applying the Fokas method for boundary value problems. Since the differential 1 -form $W(x, y, k)$ is closed, we know that

$$
\int_{\partial \Omega} e^{\left(\omega_{1}(k) x+\omega_{2}(k) y\right) \hat{\sigma}_{3}} W(x, y, k)=0
$$

The above integral can be evaluated explicitly and we find the following global relation

$$
\int_{0}^{\infty} e^{\omega_{1}(k) x \hat{\sigma}_{3}} Q(x, 0, k) \mu(x, 0, k) d x=i \int_{0}^{\infty} e^{\omega_{2}(k) y \hat{\sigma}_{3}} Q(0, y,-k) \mu(0, y, k) d y .
$$

Taking $\mu=\mu_{3}$ and using (3.2), equation (3.10) yields

$$
I-S_{1}(k)=\left(I-S_{2}(k)\right) S_{2}^{-1} S_{1}(k),
$$

which implies that $S_{2}^{-1}(k) S_{1}(k)=I$ for $k \in\left(D_{3}, D_{1}\right)$. Therefore, we obtain the global relation in terms of the spectral functions

$$
a_{1}(k)=a_{2}(k), \quad b_{1}(k)=b_{2}(k), \quad k \in D_{3} .
$$

Furthermore, substituting $S_{2}^{-1}(k) S_{1}(k)=I$ into equation (3.2), we know that $\mu_{3}(x, y, k)=\mu_{1}(x, y, k)$ for $k \in\left(D_{3}, D_{1}\right)$ and hence, we find

$$
\begin{array}{ll}
\mu_{1}^{(23)}(x, y, k)=\mu_{3}^{(34)}(x, y, k), & k \in D_{3}, \\
\mu_{1}^{(14)}(x, y, k)=\mu_{3}^{(12)}(x, y, k), & k \in D_{1} .
\end{array}
$$




\subsection{Spectral analysis at boundary values}

In this section we discuss the spectral analysis for the Lax pair at $x=0$ and $y=0$, respectively, so that the boundary values can be characterized from the spectral functions.

Proposition 3.1. The inverse map

$$
\left\{a_{1}(k), b_{1}(k)\right\} \rightarrow\left\{q(x, 0), q_{y}(x, 0)\right\}
$$

to the map defined in Definition 3.1 is given by

$$
\begin{aligned}
& \cosh q(x, 0)=1-8 i \lim _{k \rightarrow \infty} k M_{11 x}^{(x)}-8 \lim _{k \rightarrow \infty}\left(k M_{21}^{(x)}\right)^{2}, \\
& i q_{x}(x, 0)+q_{y}(x, 0)=-4 i \lim _{k \rightarrow \infty} k M_{21}^{(x)},
\end{aligned}
$$

where $M^{(x)}$ is the solution of the matrix Riemann-Hilbert problem:

$$
M_{-}^{(x)}(x, k)=M_{+}^{(x)}(x, k) J^{(x)}(x, k), \quad k \in \mathbb{R},
$$

with the jump matrix $\boldsymbol{J}^{(x)}$ given by

$$
J^{(x)}(x, k)=\left(\begin{array}{cc}
1 & \frac{b_{1}(-k)}{a_{1}(k)} e^{-2 \omega_{1}(k) x} \\
\frac{b_{1}(k)}{a_{1}(-k)} e^{2 \omega_{1}(k) x} & \frac{1}{a_{1}(k) a_{1}(-k)}
\end{array}\right), \quad k \in \mathbb{R} .
$$

Proof. The proof is based on the spectral analysis to equation (3.1a) with $y=0$ :

$$
\mu_{3}(x, 0, k)=\mu_{2}(x, 0, k) e^{-\omega_{1}(k) x \hat{\sigma}_{3}} S_{1}(k), \quad k \in\left(\mathbb{R}^{+}, \mathbb{R}^{-}\right), \quad 0 \leq x<\infty .
$$

Note that the eigenfunction $\mu_{2}^{(1)}(x, 0, k)$ is analytic and bounded for $k \in D_{1} \cup D_{2}$ and the function $\mu_{2}^{(3)}(x, 0, k)$ is analytic and bounded for $k \in D_{3} \cup D_{4}$. Thus, we formulate the matrix Riemann-Hilbert problem (3.15) with the jump matrix $J^{(x)}(x, k)$ given by (3.16), where the sectionally meromorphic functions $M_{ \pm}^{(x)}$ are defined by

$$
\begin{array}{ll}
M_{+}^{(x)}(x, k)=\left(\frac{\mu_{2}^{(1)}(x, 0, k)}{a_{1}(-k)}, \mu_{3}^{(12)}(x, 0, k)\right), & \operatorname{Im} k>0, \\
M_{-}^{(x)}(x, k)=\left(\mu_{3}^{(34)}(x, 0, k), \frac{\mu_{2}^{(3)}(x, 0, k)}{a_{1}(k)}\right), \quad \operatorname{Im} k<0 .
\end{array}
$$

Note that $\operatorname{det} M_{ \pm}^{(x)}=1$ and $M_{ \pm}^{(x)}=I+O(1 / k)$ as $k \rightarrow \infty$. Thus, we expand the solution $M^{(x)}$ of the Riemann-Hilbert problem as

$$
M^{(x)}(x, k)=I+\frac{M^{(1)}(x)}{k}+\frac{M^{(2)}(x)}{k^{2}}+O\left(1 / k^{2}\right), \quad k \rightarrow \infty .
$$

Substituting this expansion into the $x$-part of the Lax pair (2.1a) with $y=0$, from the $(2,1)$ component at $O(1)$, we find

$$
i q_{x}(x, 0)+q_{y}(x, 0)=-4 i M_{21}^{(1)}(x)
$$


and the $(1,1)$-component at $O(1 / k)$ yields

$$
M_{11 x}^{(1)}(x)=-\frac{1}{8 i}(\cosh q(x, 0)-1)-\frac{1}{4}\left(i q_{x}(x, 0)+q_{y}(x, 0)\right) M_{21}^{(1)}(x) .
$$

Simplifying the above equation with (3.20), we obtain

$$
\cosh q(x, 0)=1-8 i M_{11 x}^{(1)}(x)-8\left(M_{21}^{(1)}(x)\right)^{2} .
$$

and hence equations (3.14) are proved.

Proposition 3.2. The inverse map

$$
\left\{a_{2}(k), b_{2}(k)\right\} \rightarrow\left\{q(0, y), q_{x}(0, y)\right\}
$$

to the map defined in Definition 3.2 is given by

$$
\begin{aligned}
& \cosh q(0, y)=1+8 \lim _{k \rightarrow \infty} k M_{11 y}^{(y)}+8 \lim _{k \rightarrow \infty}\left(k M_{21}^{(y)}\right)^{2}, \\
& i q_{x}(0, y)+q_{y}(0, y)=-4 i \lim _{k \rightarrow \infty} k M_{21}^{(y)},
\end{aligned}
$$

where $M^{(y)}$ is the solution of the matrix Riemann-Hilbert problem:

$$
M_{-}^{(y)}(y, k)=M_{+}^{(y)}(y, k) J^{(y)}(y, k), \quad k \in i \mathbb{R}
$$

with the jump matrix $J^{(y)}$ given by

$$
J^{(y)}(y, k)=\left(\begin{array}{cc}
1 & \frac{b_{2}(-k)}{a_{2}(k)} e^{-2 \omega_{2}(k) y} \\
\frac{b_{2}(k)}{a_{2}(-k)} e^{2 \omega_{2}(k) y} & \frac{1}{a_{2}(k) a_{2}(-k)}
\end{array}\right), \quad k \in i \mathbb{R} .
$$

Proof. The proof is similar to that of Proposition 3.1. From the spectral relation (3.1b) with $x=0$, we find the jump matrix $J^{(y)}(y, k)$ given in (3.26) and the sectionally meromorphic functions $M_{ \pm}^{(y)}$ given by

$$
\begin{array}{ll}
M_{+}^{(y)}(y, k)=\left(\frac{\mu_{2}^{(1)}(0, y, k)}{a_{2}(-k)}, \mu_{1}^{(14)}(0, y, k)\right), & \operatorname{Re} k>0, \\
M_{-}^{(y)}(y, k)=\left(\mu_{1}^{(23)}(0, y, k), \frac{\mu_{2}^{(3)}(0, y, k)}{a_{2}(k)}\right), & \operatorname{Re} k<0
\end{array}
$$

with $\operatorname{det} M_{ \pm}^{(y)}=1$ and $M_{ \pm}^{(y)}=I+O(1 / k)$ as $k \rightarrow \infty$. Note that the eigenfunction $\mu_{2}^{(1)}(0, y, k)$ is analytic and bounded for $k \in D_{1} \cup D_{4}$ and the function $\mu_{2}^{(3)}(0, y, k)$ is analytic and bounded for $k \in$ $D_{2} \cup D_{3}$. In the similar way presented in the proof of Proposition 3.1, equations (3.24) follow. 


\section{Riemann-Hilbert problem}

Using equations (3.1), the global relation (3.11) and (3.12), we formulate the following matrix Riemann-Hilbert problem:

$$
M_{-}(x, y, k)=M_{+}(x, y, k) J(x, y, k), \quad k \in \mathscr{L},
$$

where the oriented contours $\mathscr{L}=L_{1} \cup L_{2} \cup L_{3} \cup L_{4}$ are given by (cf. Fig. 2)

$$
\begin{array}{ll}
L_{1}=D_{1} \cap D_{2}, & L_{2}=D_{2} \cap D_{3}, \\
L_{3}=D_{3} \cap D_{4}, & L_{4}=D_{4} \cap D_{1},
\end{array}
$$

and the jump matrices are defined by

$$
\begin{aligned}
& J_{1}(x, y, k)=\left(\begin{array}{cc}
1 & 0 \\
\frac{b_{2}(k)}{a_{1}(-k)} e^{2 \theta(x, y, k)} & 1
\end{array}\right), \quad k \in L_{1}, \\
& J_{2}(x, y, k)=\left(\begin{array}{rr}
-\frac{b_{1}(-k)}{a_{1}(k)} e^{-2 \theta(x, y, k)} & 1 \\
1 & 0
\end{array}\right), \quad k \in L_{2}, \\
& J_{3}(x, y, k)=\left(\begin{array}{rr}
-\frac{b_{2}(-k)}{a_{1}(k)} e^{-2 \theta(x, y, k)} & 1 \\
1 & 0
\end{array}\right), \quad k \in L_{3}, \\
& J_{4}(x, y, k)=J_{1} J_{2}^{-1} J_{3}=\left(\begin{array}{rr}
1 & 0 \\
\frac{b_{1}(k)}{a_{1}(-k)} e^{2 \theta(x, y, k)} & 1
\end{array}\right), \quad k \in L_{4}
\end{aligned}
$$

with $\theta(x, y, k)=\omega_{1}(k) x+\omega_{2}(k) y$. The matrix-valued functions $M_{ \pm}$are sectionally meromorphic and defined below

$$
\begin{aligned}
& M_{+}(x, y, k)= \begin{cases}\left(\frac{\mu_{2}^{(1)}}{a_{1}(-k)}, \mu_{1}^{(14)}\right), & k \in D_{1}, \\
\left(\mu_{1}^{(23)}, \frac{\mu_{2}^{(3)}}{a_{1}(k)}\right), & k \in D_{3},\end{cases} \\
& M_{-}(x, y, k)= \begin{cases}\left(\mu_{1}^{(23)}, \mu_{3}^{(12)}\right), & k \in D_{2}, \\
\left(\mu_{3}^{(34)}, \mu_{1}^{(14)}\right), & k \in D_{4} .\end{cases}
\end{aligned}
$$

Note that $\operatorname{det} M_{ \pm}=1$ and $M_{ \pm}=I+O(1 / k)$ as $k \rightarrow \infty$. The Riemann-Hilbert problem (4.1) can be solved by a Cauchy-type integral equation. Indeed, letting $\tilde{J}=I-J$, equation (4.1) becomes

$$
M_{+}(x, y, k)-M_{-}(x, y, k)=M_{+}(x, y, k) \tilde{J}(x, y, k) .
$$

Applying the Plemelj formula [9], the solution $M$ of the Riemann-Hilbert problem (4.1) can be expressed as

$$
M(x, y, k)=I+\frac{1}{2 i \pi} \int_{\mathscr{L}} M_{+}\left(x, y, k^{\prime}\right) \tilde{J}\left(x, y, k^{\prime}\right) \frac{d k^{\prime}}{k^{\prime}-k}
$$

Note that

$$
M(x, y, k)=I-\frac{1}{2 i k \pi} \int_{\mathscr{L}} M_{+}\left(x, y, k^{\prime}\right) \tilde{J}\left(x, y, k^{\prime}\right) d k^{\prime}+O\left(1 / k^{2}\right), \quad k \rightarrow \infty .
$$


Then the solution of the elliptic sinh-Gordon equation in the quarter plane can be obtained in terms of the unique solution of the Riemann-Hilbert problem. In this respect, we expand the solution $M$ of the Riemann-Hilbert problem (4.1) as

$$
M(x, y, k)=I+\frac{M^{(1)}(x, y)}{k}+\frac{M^{(2)}(x, y)}{k^{2}}+O\left(1 / k^{2}\right), \quad k \rightarrow \infty .
$$

Substituting this expansion into the $x$-part of the Lax pair $(2.1 \mathrm{a})$, the $(2,1)$-component at $O(1)$ implies

$$
i q_{x}(x, y)+q_{y}(x, y)=-4 i M_{21}^{(1)}(x, y)
$$

and the $(1,1)$-component at $O(1 / k)$ yields

$$
M_{11 x}^{(1)}(x, y)=-\frac{1}{8 i}(\cosh q(x, y)-1)-\frac{1}{4}\left(i q_{x}(x, y)+q_{y}(x, y)\right) M_{21}^{(1)}(x, y) .
$$

Simplifying the above equation with (4.7), we obtain the reconstruction formula for the solution of (1.1) given by

$$
\cosh q(x, y)=1-8 i M_{11 x}^{(1)}(x, y)-8\left(M_{21}^{(1)}\right)^{2} .
$$

Similarly, if we substitute the expansion (4.6) into the $y$-part of the Lax pair, the solution is equivalently given by

$$
\cosh q(x, y)=1+8 M_{11 y}^{(1)}(x, y)+8\left(M_{21}^{(1)}\right)^{2} .
$$

We now state the existence theorem for the elliptic sinh-Gordon equation in the quarter plane.

Theorem 4.1. Assume that the functions $g_{j}(x), f_{j}(y) \in H^{1}\left(\mathbb{R}^{+}\right), j=0,1$, with the sufficiently small $H^{1}$ norms. Let the functions $a_{1}(k), b_{1}(k), a_{2}(k)$ and $b_{2}(k)$ be given by (3.5) and (3.8) in Definitions 3.1 and 3.2, respectively. Suppose that given $g_{0}(x)$ and $g_{1}(x)$, there exist functions $f_{0}(y)$ and $f_{1}(y)$ such that the global relation is satisfied

$$
a_{1}(k)=a_{2}(k) \quad \text { and } \quad b_{1}(k)=b_{2}(k) \quad k \in D_{3} .
$$

Let $M(x, y, k)$ be the solution of the following matrix Riemann-Hilbert $(R H)$ problem

$$
M_{-}(x, y, k)=M_{+}(x, y, k) J(x, y, k), \quad k \in \mathscr{L},
$$

where $\operatorname{det}\left(M_{ \pm}\right)=1, M_{ \pm}=I+O(1 / k)$ as $k \rightarrow \infty$, the oriented contours $\mathscr{L}$ are defined in (4.2) and the jump matrices $J$ are given in (4.3).

Then the Reimann-Hilbert problem is uniquely solvable and the function $q(x, y)$ defined by

$$
i q_{x}+q_{y}=-4 i \lim _{k \rightarrow \infty} k M_{21}, \quad \cosh q(x, y)=1-8 i \lim _{k \rightarrow \infty} k M_{11 x}-8 \lim _{k \rightarrow \infty}\left(k M_{21}\right)^{2}
$$

solves the elliptic sinh-Gordon equation (1.1) satisfying the boundary conditions

$$
\begin{array}{lll}
q(x, 0)=g_{0}(x), & q_{y}(x, 0)=g_{1}(x), \\
q(0, y)=f_{0}(y), & q_{x}(0, y)=f_{1}(y) .
\end{array}
$$


Proof. By applying the vanishing lemma and the dressing method discussued in [9,14], it can be proved that the Riemann-Hilbert problem (4.11) is uniquely solved and that $q(x, y)$ defined in (4.12) solves the elliptic sinh-Gordon equation (1.1) (see also before Theorem).

We will prove that $q(x, y)$ given in (4.12) satisfies the boundary values. In this respect, it requires to show that the Riemann-Hilbert problem (4.11) with $y=0$ and $x=0$ are equivalent to the Riemann-Hilbert problems (3.15) and (3.25) given in Propositions 3.1 and 3.2, respectively.

Regarding equation (4.13a), define

$$
M^{(x)}(x, k)=\left\{\begin{array}{l}
M(x, 0, k), \quad k \in D_{1}, \\
M(x, 0, k) J_{1}^{-1}(x, 0, k), \quad k \in D_{2}, \\
M(x, 0, k) J_{3}(x, 0, k) F(x, k), \quad k \in D_{3}, \\
M(x, 0, k) F(x, k), \quad k \in D_{4},
\end{array}\right.
$$

where

$$
F(x, k)=\left(\begin{array}{cc}
1 & \frac{b_{1}(-k)}{a_{1}(k)} e^{-2 \omega_{1}(k) x} \\
0 & 1
\end{array}\right) .
$$

We denote $M(x, 0, k)$ and $M^{(x)}(x, k)$ for $k \in D_{j}, j=1, \cdots, 4$, by $M_{j}(x, 0, k)$ and $M_{j}^{(x)}(x, k)$, respectively. Then, we can write (4.11) and (4.14) as

$$
\begin{array}{ll}
M_{2}(x, 0, k)=M_{1} J_{1}(x, 0, k), & M_{2}(x, 0, k)=M_{3} J_{2}(x, 0, k), \\
M_{4}(x, 0, k)=M_{3} J_{3}(x, 0, k), & M_{4}(x, 0, k)=M_{1} J_{4}(x, 0, k),
\end{array}
$$

and

$$
\begin{aligned}
& M_{1}^{(x)}(x, k)=M_{1}(x, 0, k), \quad M_{2}^{(x)}(x, k)=M_{1} J_{1}^{-1}(x, 0, k), \\
& M_{3}^{(x)}(x, k)=M_{3} J_{3}(x, 0, k) F(x, k), \quad M_{4}^{(x)}(x, k)=M_{4}(x, 0, k) F(x, k) .
\end{aligned}
$$

Combining (4.17) with (4.16), we find the following jump conditions

$$
\begin{array}{ll}
M_{2}^{(x)}(x, k)=M_{1}^{(x)}(x, k), & M_{3}^{(x)}(x, k)=M_{2}^{(x)}(x, k) J_{4}(x, 0, k) F(x, k), \\
M_{4}^{(x)}(x, k)=M_{3}^{(x)}(x, k), & M_{4}^{(x)}(x, k)=M_{1}^{(x)}(x, k) J_{4}(x, 0, k) F(x, k) .
\end{array}
$$

Note that $J_{4}(x, 0, k) F(x, k)=J^{(x)}(x, k)$, where $J^{(x)}(x, k)$ is given in (3.16), and no jumps occur along the contours $L_{1}$ and $L_{3}$. Defining

$$
\begin{array}{ll}
M^{(x)}(x, k)=M_{+}^{(x)}(x, k) & k \in D_{1} \cup D_{2}, \\
M^{(x)}(x, k)=M_{-}^{(x)}(x, k) & k \in D_{3} \cup D_{4},
\end{array}
$$

we know that equations (4.16) are equivalent to the Riemann-Hilbert problem (3.15) with the jump matrix $J^{(x)}(x, k)$. Thus, the proof that the function $q(x, y)$ satisfies the boundary values (4.13a) immediately follows from evaluating (4.12) at $y=0$.

In a similar way, equation (4.13b) can be proved. 


\section{Concluding remarks}

In conclusion, we have studied the boundary value problem for the elliptic sinh-Gordon equation formulated in the quarter plane by using the Fokas method. Based on the Lax pair formulation we have derived the global relation that involves all boundary values $\left\{q(x, 0), q_{y}(x, 0)\right\}$ and $\left\{q(0, y), q_{x}(0, y)\right\}$. Furthermore, if the boundary values satisfy the global relation, we have presented the existence of the unique solution for the elliptic sin-Gordon equation in the quarter plane. It also has been shown that the solution can be expressed in terms of the unique solution of the Riemann-Hilbert problem with the jump matrices defined by the spectral functions $\left\{a_{1}(k), b_{1}(k)\right\}$ and $\left\{a_{2}(k), b_{2}(k)\right\}$.

It should be remarked that using the global relation, we have determined the spectral functions $\left\{a_{1}(k), b_{1}(k)\right\}$ and $\left\{a_{2}(k), b_{2}(k)\right\}$ in terms of the boundary values $\left\{q(x, 0), q_{y}(x, 0)\right\}$ and $\left\{q(0, y), q_{x}(0, y)\right\}$. However, it is not necessary to prescribe all boundary values for well-posed boundary value problems. Thus, it should be required to characterize unknown boundary values, called the Dirichlet to Neumann map [8,11]. This characterization can be done by analyzing the global relation as was done in $[12,13]$ and we will discuss this issue in the near future.

\section{References}

[1] M. J. Ablowitz and P. A. Clarkson, Solitons, Nonlinear Evolution Equations and Inverse Scattering, Cambridge: Cambridge University Press, 1991.

[2] G. Biondini and G. Hwang, Initial-boundary value problems for discrete evolution equations: discrete linear Schrödinger and integrable discrete nonlinear Schrödinger equations, Inv. Probl. 24, (2008) 065011: $1-44$.

[3] M. Boiti, J. J-P. Leon and F. Pempinelli, Integrable two-dimensional generalisation of the sine- and sinhGordon equations, Inverse Problems 3 (1987) 37-49.

[4] A. S. Fokas, A unified transform method for solving linear and certain nonlinear PDEs, Proc. Roy. Soc. London A 453 (1997) 1411-1443.

[5] A. S. Fokas, On the integrability of certain linear and nonlinear partial differential equations, J. Math. Phys. 41 (2000) 4188-4237.

[6] A. S. Fokas, Two dimensional linear PDEs in a convex polygon, Proc. Roy. Soc. London A 457 (2001) 71-393.

[7] A. S. Fokas, Integrable nonlinear evolution equations on the half-line, Comm. Math. Phys. 230 (2002) $1-39$.

[8] A. S. Fokas, The generalized Dirichlet-to-Neumann map for certain nonlinear evolution PDEs, Comm. Pure Appl. Math. LVIII (2005) 639-670.

[9] A. S. Fokas, A Unified Approach to Boundary Value Problems, CBMS-NSF regional conference series in applied mathematics, SIAM 2008.

[10] A. S. Fokas and J. Lenells, The unified method: I. Non-linearizable problems on the half-line, J. Phys. A: Math. Theor. 45 (2012) 195201.

[11] A. S. Fokas and B. Pelloni, The Dirichlet-to-Nemann map for the elliptic sine-Gordon equation, Nonlinearity 25 (2012) 1011-1031.

[12] G. Hwang, The Fokas method: The Dirichlet to Neumann map for the sine-Gordon equation, Stud. Appl. Math. 132 (2014) 381-406.

[13] G. Hwang, A perturbative approach for the asymptotic evaluation of the Neumann value corresponding to the Dirichlet datum of a single periodic exponential for the NLS, J. Nonlinear Math. Phys. 21 (2014) $225-247$.

[14] G. Hwang, The elliptic sinh-Gordon equation in the half plane, J. Nonlinear Sci. and Appl. 8 (2015) $163-173$.

[15] G. Hwang and A. S. Fokas, The modified Korteweg-de Vries equation on the half-line with a sine-wave as Dirichlet datum, J. Nonlinear Math. Phys. 20 (2013) 135-157. 
[16] M. Jaworski and D. Kaup, Direct and inverse scattering problem associated with the elliptic sinhGordon equation, Inverse Problems 6 (1990) 543-556.

[17] B. Pelloni, Spectral analysis of the elliptic sine-Gordon equation in the quarter plane, Theor. Math. Phys. 160 (2009) 1031-1041.

[18] B. Pelloni and D. A. Pinotsis, The elliptic sine-Gordon equation in a half plane, Nonlinearity 23 (2010) 77-88. 